\title{
A KOROVKIN-TYPE THEOREM IN LOCALLY CONVEX $M$-SPACES
}

HANS O. FLÖSSER

\begin{abstract}
Let $E$ be a locally convex $M$-space, $\varnothing \neq M$ a subset. The universal Korovkin-closure of $M$ as well as the sequentially or stationary defined Korovkin-closures coincide with the space of $\boldsymbol{M}$-harmonic elements and with the uniqueness closure of $M$.
\end{abstract}

1. The Theorem. Let $E, F$ denote locally convex vector lattices ([6], but not necessarily separated); $L(E, F)_{+}$is the cone of continuous positive linear operators from $E$ into $F$ and $V(E, F)$ the set of all continuous linear lattice homomorphisms from $E$ into $F$. We write $E_{+}^{\prime}$ for $L(E, \mathbf{R})_{+}$and $V(E)$ for $V(E, \mathbf{R})$. For $\varnothing \neq M \subset E$ the universal Korovkin closure $K(M)([9]$, [10]) is defined by $e \in K(M)$

$$
\left\{\begin{array}{l}
\text { iff for any locally convex vector lattice } F \\
\text { and for any equicontinuous net } T_{\alpha} \in L(E, F)_{+} \\
\text {and for any } S \in V(E, F) \text { the relation } \\
\lim _{\alpha} T_{\alpha} f=S f \text { for all } f \in M \text { implies } \lim _{\alpha} T_{\alpha} e=S e .
\end{array}\right.
$$

Let $K_{\sigma}(M)$ and $K_{0}(M)$ denote the set of all $e \in E$ that satisfy (*), when in (*) the word "net" is replaced by "sequence" and "stationary sequence", respectively.

$L(M)$ is the closed linear hull of $M$. Let $\hat{M}$ be the set of all finite infima of elements in $L(M)$, i.e.

$$
\hat{M}=\{\wedge A \mid \varnothing \neq A \subset L(M), A \text { finite }\}
$$

Then the set $H(M)$ of $M$-harmonic elements is defined as $H(M)=\overline{\hat{M}} \cap$ - $\hat{M}$ ([8], [9]). Note that $-\hat{M}=\check{M}$ is the set of all finite suprema of elements of $L(M)$.

By $U(M)$ we denote the uniqueness closure of $M$, i.e. $e \in U(M)$ iff for all $\mu \in E_{+}^{\prime}, \delta \in V(E)$ equality of $\mu$ and $\delta$ on $M$ implies $\mu(e)=\delta(e)$ ([10]; cf. [2]).

A locally convex vector lattice $E$ is called a locally convex $M$-space, if its topology is generated by a family $\left\{\|\|_{\alpha}\right\}$ of lattice seminorms which satisfy $\|e \vee f\|_{\alpha}=\|e\|_{\alpha} \vee\|f\|_{\alpha}$ for all positive $e, f \in E$ ("espaces de Kakutani" in [5], cf. [7, II §7]). Such seminorms will be called $M$-seminorms in the sequel.

Received by the editors October 31, 1977 and, in revised form, April 3, 1978.

AMS (MOS) subject classifications (1970). Primary 46A40; Secondary 46E05.

- American Mathematical Society 1978 
TheOREM. Let $E$ be a locally convex $M$-space and $\varnothing \neq M$ a subset. Then $H(M)=K(M)=K_{\sigma}(M)=K_{0}(M)=U(M)$.

Before proving the theorem let us compare its statement with results obtained by other authors.

When $E=C(X), X$ a compact metric space, and $M$ is a point-separating subset containing a strict positive function, the equality $K_{o}(M)=U(M)$ was proved by H. Berens and G. G. Lorenz in [2]. Since here $M$ is an arbitrary subset of $E$, we have a new result even in this case.

The equality $H(M)=K(M)$ was proved by M. Wolff in [9] for locally convex vector lattices, if the closed linear hull $L(M)$ of $M$ is nearly positively generated in the sense that $L(M)=\overline{L(M)_{+}-L(M)_{+}}$. If in addition $E$ is dual atomic (i.e. $E^{\prime}$ is atomic), then $K(M)=U(M)$ as was proved also by M. Wolff in [10]. Since locally convex $M$-spaces are not dual atomic in general, Wolff's theorem and our theorem cover different cases.

As a class of locally convex lattices where our theorem could be applied, one has the class of so-called weighted-function spaces $C V_{0}(X)$, which have been studied by many authors ([3]). They include well-known spaces as for example $E=C(X), X$ completely regular, equipped with the topology of compact convergence or $E=C B(X)$, the bounded continuous real functions on $X$, with the strict topology.

2. Proof of the Theorem. An essential tool in proving the Theorem is the concept of upper and lower envelopes of elements $e \in E$. This concept in connection with Korovkin-theorems is not new: it has been used already by H. Bauer ([1]), H. Berens and G. G. Lorenz ([2]) and by K. Donner ([4]).

Let $E$ be a vector lattice equipped with a lattice seminorn \|\|$(|e| \leqslant|f|$ implies $\|e\| \leqslant\|f\|$ ), $E^{\prime}$ its (topological) dual and $B$ the positive part of the unit ball of $E^{\prime}$. In the weak topology $\sigma\left(E^{\prime}, E\right)$ the set $B$ is compact. The evaluation map $E \rightarrow A_{0}(B)$ sends elements $e \in E$ in continuous affine functions $\tilde{e}$ on $B$ vanishing at $0 \in B$. Therefore we can define upper and lower envelopes of $e \in E$ as

$$
\begin{aligned}
& \hat{e}(\mu)=\inf \{\mu(f)+r \mid f \in \hat{M}, r \in \mathbf{R}, \tilde{f}+r \geqslant \tilde{e}\}, \\
& \check{e}(\mu)=\sup \{\mu(f)+r \mid f \in \check{M}, r \in \mathbf{R}, \tilde{f}+r \leqslant \tilde{e}\}
\end{aligned}
$$

for all $\mu \in B$ (pointwise order on $B$ ). ${ }^{1}$

We collect some simple properties of the envelopes in the following lemma:

Lemma 1. Let $e \in E, \mu \in B$.

(i) $\check{e}(\mu) \leqslant \mu(e) \leqslant \hat{e}(\mu)$ with equality on $L(M)$.

(ii) $(-e)^{\wedge}(\mu)=-\check{e}(\mu)$.

(iii) $e \leqslant 0$ implies $\hat{e}(\mu) \leqslant 0$.

\footnotetext{
'To use this kind of envelope was suggested by K. Donner at the June 1977 meeting on "Riesz spaces and order bounded linear transformations" in Oberwolfach.
} 
(iv) $\hat{e}(\mu) \leqslant\|e\|$.

(v) The map $e \mapsto \hat{e}(\mu)$ is a sublinear functional on $E$.

We omit the easy proof.

Now, let $V(E)_{1}=V(E) \cap B$ and denote by $E(M)$ the set of all $e \in E$ the upper and lower envelopes of which coincide on $V(E)_{1}$.

Lemma 2. Let $e \in E, \delta \in B$. Then there exists $a \mu \in B$ such that $\mu(e)=$ $\hat{e}(\delta)$ and $\mu={ }_{M} \delta$.

Proof. By (v) of Lemma 1 the mapping $p_{\delta}: f \mapsto \hat{f}(\delta)$ is a sublinear functional on $E$. The linear functional $\mu_{0}$ on $\mathbf{R} \cdot e$ defined by $\mu_{0}(r e)=r \hat{e}(\delta)$ is dominated by $p_{\delta}$; this is evident for $r \geqslant 0$. For $r<0$ it follows by $\mu_{0}(-e)=$ $-\hat{e}(\delta) \leqslant-\delta(e)=\delta(-e) \leqslant(-e)^{\wedge}(\delta)=p_{\delta}(-e)$ using (i) of Lemma 1.

The Hahn-Banach theorem yields an extension $\mu$ of $\mu_{0}$ dominated by $p_{\delta}$ on $E$. By (iv) of Lemma $1, \mu$ is continuous with norm $\leqslant 1$. By (iii) of Lemma 1 it is positive and thus belongs to $B$. Finally $\mu \leqslant p_{\delta}$ implies $\mu={ }_{M} \delta$ as $\delta(f)=$ $\hat{f}(\delta)$ on $L(M)$.

LEMMA 3. We have $U(M)=E(M)$.

Proof. Suppose $e$ belongs to $U(M)$ and let $\delta \in V(E)_{1}$. By Lemma 2 there exists a $\mu \in B$ such that $\mu(e)=\hat{e}(\delta)$ and $\mu={ }_{M} \delta$. As $e \in U(M), \mu(e)=$ $\delta(e)$ and $\delta(e)=\hat{e}(\delta)$. Since also $-e \in U(M), \delta(-e)=-\delta(e)=(-e)^{\wedge}(\delta)$ $=-\check{e}(\delta)$ and $\delta(e)=\check{e}(\delta)$. Thus $e$ belongs to $E(M)$.

Conversely, let $e \in E(M)$ and choose $\mu \in E_{+}^{\prime}, \delta \in V(E)$ such that $\mu$ $={ }_{M} \delta$. By multiplying by a positive constant, if necessary, we can assume $\mu \in B, \delta \in V(E)_{1}$. Now let $f \in \hat{M}$ and write $f=\bigvee_{i=1}^{n} f_{i}$ with $f_{i} \in L(M)$ $(i=1, \ldots, n)$. Since $\delta$ is a lattice homomorphism and since $\mu={ }_{M} \delta$, it follows that

and

$$
\delta(f)=\bigvee_{i=1}^{n} \delta\left(f_{i}\right)=\bigvee_{i=1}^{n} \mu\left(f_{i}\right) \leqslant \mu(f)
$$

$$
\delta(f)+r \leqslant \mu(f)+r \text { for all } r \in \mathbf{R} .
$$

The definition of lower envelopes yields $\check{e}(\delta) \leqslant \check{e}(\mu)$. Similarly, one obtains $\hat{e}(\mu) \leqslant \hat{e}(\delta)$. Thus $e \in E(M)$ implies-using (i) of Lemma $1-\mu(e)=\delta(e)$ and $e \in U(M)$.

LEMMA 4. Let $E$ have an $M$-seminorm. If $e \in E$ satisfies $\delta(e)=\hat{e}(\delta)$ for all $\delta \in V(E)_{1}$, then $e \in \hat{\bar{M}}$.

Proof. First observe that $\tilde{e} \leqslant \tilde{f}+r, r \in \mathbf{R}$, implies $r \geqslant 0$ since $0 \in B$. Suppose $\varepsilon>0, \delta \in V(E)_{1}$, by hypothesis on $e$ there exist $f \in \hat{M}, 0 \leqslant r \in \mathbf{R}$, such that $\tilde{e} \leqslant \tilde{f}+r$ and

$$
\left(\frac{1}{2} \delta\right)(f)+r<\hat{e}\left(\frac{1}{2} \delta\right)+\frac{1}{2} \varepsilon=\frac{1}{2}(\delta(e)+\varepsilon) \leqslant \frac{1}{2}(\delta(f)+r+\varepsilon) .
$$

Thus $0 \leqslant r<\varepsilon, \tilde{e} \leqslant \tilde{f}+\varepsilon$ and $\delta(f)<\delta(e)+\varepsilon$. Hence the sets $U_{f}=\{\delta \in$ 
$\left.V(E)_{1} \mid \delta(f)<\delta(e)+\varepsilon\right\}$ form a $\sigma\left(E^{\prime}, E\right)$-open covering of the $\sigma\left(E^{\prime}, E\right)$ compact set $V(E)_{1}$, when $f$ varies in $\hat{M}$ such that $\tilde{e} \leqslant \hat{f}+\varepsilon$. We therefore find finitely many $f_{1}, \ldots, f_{n} \in \hat{M}$ such that $\tilde{e} \leqslant \tilde{f}_{i}+\varepsilon$ for all $i=1, \ldots, n$ and $V(E)_{1}=\cup_{i=1}^{n} U_{f_{i}}$.

Let $f=\bigwedge_{i=1}^{n} f_{i}$; then $f \in \hat{M}$ and for an arbitrary $\delta \in V(E)_{1}$ we have

$$
-\varepsilon+\delta(e) \leqslant \delta(f)=\min _{i=1, \ldots, n} \delta\left(f_{i}\right)<\delta(e)+\varepsilon .
$$

Thus $\sup _{\delta \in V(E)_{1}}|\delta(f-e)| \leqslant \varepsilon$. Since $E$ has an $M$-seminorm, $V(E)_{1}$ contains the extreme points of $B$, so that $\sup _{\delta \in V(E)}|\delta(f-e)|=\|f-e\| \leqslant \varepsilon$. As $\varepsilon>0$ was arbitrary, $e \in \overline{\hat{M}}$ as required.

Now we are able to prove the Theorem.

Proof of THE TheOREM. The inclusion $H(M) \subset K(M)$ was proved by M. Wolff in [8] for arbitrary locally convex vector lattices. The inclusions $K(M) \subset K_{\sigma}(M) \subset K_{0}(M) \subset U(M)$ follow immediately by the definitions of the respective spaces.

To prove $U(M) \subset H(M)$ we proceed as follows. Let $\left\{\|\|_{\alpha}\right\}_{\alpha \in A}$ be a saturated family of $M$-seminorms generating the topology of $E$ and denote by $E_{\alpha}$ the space $E$ seminormed by \|\|$_{\alpha}$. Furthermore let $U_{\alpha}(M), E_{\alpha}(M)$ and $H_{\alpha}(M)$ denote the spaces $U(M), E(M)$ and $H(M)$ constructed in $E_{\alpha}$. By Lemma 3 we have $U_{\alpha}(M)=E_{\alpha}(M)$, by Lemma 4 and (ii) of Lemma 1, $E_{\alpha}(M) \subset H_{\alpha}(M)$. Now the assertion of the Theorem follows by

$$
U(M) \subset \bigcap_{\alpha \in A} U_{\alpha}(M)=\bigcap_{\alpha \in A} E_{\alpha}(M) \subset \bigcap_{\alpha \in A} H_{\alpha}(M)=H(M) .
$$

In [10] M. Wolff proved $U(M)=K(M, I)$ for the identity Korovkin closure $K(M, I)$ of $M$ in $E$ (i.e. in the definition (*) only $F=E$ and $S=I$, the identity on $E$, is allowed), if $E$ is a locally convex $M$-space. Thus our theorem together with Wolff's result implies:

COROLlary. If $E$ is a locally convex $M$-space, then the identity Korovkin closure of $M$ in $E$ is universal and coincides both with the set of $M$-harmonic elements and the uniqueness closure of $M$.

\section{REFERENCES}

1. H. Bauer, Theorems of Korookin type for adapted spaces, Ann. Inst. Fourier (Grenoble) 23 (1973), 245-260.

2. H. Berens and G. G. Lorentz, Theorems of Korovkin type for positive linear operators on Banach lattices, Approximation Theory (G. G. Lorentz, ed.), Academic Press, New York, 1973.

3. K.-D. Bierstedt, Gewichtete Räume stetiger vektorwertiger Funktionen und das injektive Tensorprodukt. I, J. Reine Angew. Math. 259 (1973), 186-210.

4. K. Donner, Korookin theorems for positive linear operators, J. Approximation Theory 13 (1975), 443-450.

5. C. Portenier, Espaces de Riesz, espaces de fonctions et espaces de sections, Comment. Math. Helv. 46 (1971), 289-313.

6. H. H. Schaefer, Topological vector spaces, Springer-Verlag, Berlin and New York, 1974.

7. ___ Banach lattices and positive operators, Springer-Verlag, Berlin and New York, 1974. 
8. M. Wolff, Über die Korookinhülle von Teilmangen in lokalkonvexen Vektorverbänden, Math. Ann. 213 (1975), 97-108.

9. _ On the universal Korovkin closure of subsets in vector lattices, J. Approximation Theory 22 (1978), 243-253.

10. $\quad$ On the theory of approximation by positive operators in vector lattices, Functional Analysis: Surveys and Recent Results (K.-D. Bierstedt, B. Fuchssteiner, eds.), North-Holland Mathematics Studies, vol. 27, Amsterdam, 1977.

Fachiereich Mathematix, Technische Hochschule, D-6100 Darmstadt, Federal RepUbLIC OF GERMANY 